\title{
Knockdown of TRB3 induces apoptosis in human lung adenocarcinoma cells through regulation of Notch 1 expression
}

\author{
HUI ZHOU ${ }^{1,2}$, YI LUO ${ }^{1}$, JIAN-HUA CHEN ${ }^{1}$, JUN HU ${ }^{1}$, YONG-ZHONG LUO ${ }^{1}$, \\ WEI WANG ${ }^{1}$, YONG ZENG ${ }^{1,3}$ and LING XIAO ${ }^{4}$ \\ ${ }^{1}$ Tumor Hospital of Xiangya School of Medicine; ${ }^{2}$ Oncology Department of Xiangya Hospital; \\ ${ }^{3}$ State Key Laboratory of Medical Genetics; ${ }^{4}$ Department of Histology and Embryology of School of Basic Medical Sciences, \\ Central South University, Changsha, Hunan 410013, P.R. China
}

Received February 9, 2013; Accepted April 25, 2013

DOI: $10.3892 / \mathrm{mmr} .2013 .1453$

\begin{abstract}
The upregulation of tribbles homolog 3 (TRB3), a pseudokinase in mammals, has been observed in several types of malignant cancer, including thyroid, ovarian, liver and colorectal cancer. However, the pathological role and the regulatory mechanism of TRB3 in cancer remain unknown. In the current study, we demonstrated that the expression of TRB3 was upregulated in non-small cell lung cancer (NSCLC), correlating with tumor metastasis, disease recurrence and poor survival in patients. Knocking down TRB3 in aggressive lung cancer cell lines was demonstrated to significantly inhibit their malignant behaviors, including in vitro invasion and cell proliferation, as well as in vivo metastasis and tumor growth. The correlation between TRB3 and Notch 1 expression revealed that Notch 1 was downregulated by the knockdown of TRB3 in the lung adenocarcinoma cell lines. These results have provided insights into the correlation between TRB3 expression and lung cancer progression, and thus may have potential for the prognosis and therapy of lung cancer.
\end{abstract}

\section{Introduction}

Tribbles homolog 3 (TRB3), a pseudokinase, is essential for the catalytic activity that is performed by $10 \%$ of the kinase superfamily members (1). TRB3 was also demonstrated to be a key factor in complementary kinome small interferingRNA (siRNA) function, as a regulator of mitogen-activated protein kinase (MAPK) signaling (2). Previous studies have demonstrated that this occurs through the control of the MAPK-extracellular signal-related kinase (MAPK-ERK) and transforming growth factor $\beta$ (TGF $\beta$ ) pathways.

Correspondence to: Dr Ling Xiao, Department of Histology and Embryology of School of Basic Medical Sciences, Central South University, 172 Tongzipo Road, Changsha, Hunan 410013, P.R. China

E-mail: lingxiaocsu@126.com

Key words: tribbles homolog 3, Notch 1, non-small cell lung cancer, A549, lung cancer
Furthermore, TRB3 regulates JAG1 expression and is required for the proliferation of breast cancer cells (3). In addition, TRB3 is required in normal tissues during conditions of hypoxic/endoplasmic reticulum stress or nutrient deprivation, as it is upregulated and counteracts the effects of stress $(4,5)$. TRB3 is also upregulated in cancer as a response to hypoxia $(4,6)$ and is associated with a poor outcome $(7)$ as it promotes the activation of key cancer signaling pathways (such as MAPK-ERK, TGF $\beta$ and jagged 1 protein (JAG1)/Notch). TRB3 expression and molecular function has rarely been demonstrated in cancer cell lines, with the exception of breast cancer cell lines (2).

The most understood function of the Notch family is cell fate regulation. This function has been regarded to be linked to the homeostasis of stem cell compartments (8-11) and thus, Notch signaling has been implicated in human cancer (10). Cell-autonomous oncogenic activation of Notch was identified in T-cell acute lymphoblastic leukemia/lymphoma (T-ALL). Notch 1 may be activated through chromosomal translocations and/or mutations $(10,12)$. Downregulated expression of Notch-related factors, including Notch receptors, ligands and targets, has also been observed in solid tumors $(10,13)$, including breast (13) and lung (14) cancer. To the best of our knowledge, no studies with regard to the correlation between cell-autonomous activation of Notch and TRB3 expression in lung cancer have been published to date. Furthermore, the loss of NUMB expression in breast cancer may contribute to increased Notch activity and Notch-dependent proliferation $(15,16)$.

JAG1/Notch signaling has been considered to be a mediator of cancer progression and metastasis associated with the basal-like subtype $(17,18)$. The majority of lung cancer patients have basal-like disease, and despite initial responses to systemic cytotoxic chemotherapy, the disease follows an aggressive clinical course with early recurrence (19). Therefore, JAG1/Notch signaling and regulators of this pathway are attractive therapeutic targets in this lung cancer subtype. TRB3 influences the tumor cell biology and may be regulated by the JAG1/Notch pathways. Tumor-initiating cells represent a small population of cells within certain types of tumors, which possess the unique ability to self-renew and to produce derivatives that maintain the tumor. The TRB3 target 
pathways, including Notch, MAPK-ERK and TGFß $(20,21)$, have been implicated in tumor-initiating cell maintenance, suggesting that through the control of these pathways, TRB3 may regulate the initiation of tumor formation. The metastatic potential of epithelial tumors is likely to depend on a process known as epithelial-to-mesenchymal (EMT) transition, where epithelial cells acquire a migratory mesenchymal phenotype (22). The Notch, TGF $\beta$ and MAPK-ERK pathways interact and have a synergistic effect on the production of factors that promote EMT and metastasis (23-25). The Notch and TGF $\beta$ pathways have been demonstrated to facilitate metastasis, and also have a role in determining the location of metastatic sites (26); TGF $\beta$ released from bone metastases induces JAG1 expression in tumor cells, which contributes to paracrine Notch activation in osteoblasts and preosteoclasts, and thus leads to bone invasion. This suggests that TRB3 may potentiate the initiation of tumor formation and the metastatic capacity of lung cancer cells through the regulation of JAG1/ Notch activation. Additionally, the activation of these pathways and processes may result in reduced survival associated with tumors, and elevated TRB3 levels.

We hypothesized that the abnormal expression of TRB3 may participate in lung cancer development. By transfection analysis, we demonstrated the effect of knocking down TRB3 on human lung adenocarcinoma cells and the underlying molecular mechanism. The aim of the current study was to investigate the therapeutic potential of the knockdown of TRB3 in lung cancer.

\section{Materials and methods}

Reagents and antibodies. Rabbit antibodies against human TRB3 (T8076) and Notch (SAB2101618) were purchased from Sigma-Aldrich (St. Louis, MO, USA). Mouse antibody against human $\beta$-actin (sc-8432) and the secondary antibodies conjugated with horseradish peroxidase against mouse and rabbit IgG (sc-2005 and sc-2030, respectively) were purchased from Santa Cruz Biotechnology, Inc. (Santa Cruz, CA, USA).

Clinical specimens, cells, plasmids and transfection. Clinical samples for quantitative PCR (Q-PCR) and immunohistochemistry (IHC) were obtained from Xiangya School of Medicine, Central South University (Changsha, Hunan, China) with informed patient consent and approval of the institutional review board (Xiangya School of Medicine Research Ethics Committee). Human lung adenocarcinoma cell lines were obtained from the American Type Culture Collection (ATCC, Manassas, VA, USA). The recombinant expression plasmid of pcDNA3.1(t) (pc3.1) expressing TRB3 was constructed. Briefly, the open reading frame of TRB3 (GenBank accession: $\mathrm{BC} 027484)$ was cloned into plasmid pcDNA3.1(t) (Invitrogen Life Technologies, Carlsbad, CA, USA) between the XhoI and BamHI sites to build the pc3.1-shTRB3 recombinant plasmid. The A549 cells were cultured in Dulbecco's modified Eagle's medium (DMEM; Gibco-BRL, Carlsbad, CA, USA) supplemented with $10 \%$ fetal bovine serum (FBS; Gibco-BRL) at $37^{\circ} \mathrm{C}$ in an incubator with an atmosphere of $5 \% \mathrm{CO}_{2}$. The cells were transfected with pc3.1-shTRB3 using Lipofectamine ${ }^{\mathrm{TM}} 2000$ (Invitrogen Life Technologies) according to the manufacturer's instructions.
Semi-quantitative RT-PCR. Total RNAs were isolated using TRIzol reagent (Invitrogen Life Technologies), according to the manufacturer's instructions. The first-strand complementary DNA (cDNA) was reverse transcribed from $2 \mu \mathrm{g}$ RNA in a final volume of $20 \mathrm{ml}$, using SuperScript II Reverse Transcriptase (Invitrogen Life Technologies). The primers were designed in accordance with GenBank. The quantity of cDNA used for each PCR reaction was $20 \mathrm{ng}$ in a $50 \mathrm{ml}$ reaction volume. The PCR was performed with the Applied Biosystems 7500 RealTime PCR system (Invitrogen Life Technologies). The protocol was as follows: one cycle at $94^{\circ} \mathrm{C}$ for $4 \mathrm{~min}$ and 40 cycles at $94^{\circ} \mathrm{C}$ for $30 \mathrm{sec}, 60^{\circ} \mathrm{C}$ for $30 \mathrm{sec}$ and $72^{\circ} \mathrm{C}$ for $30 \mathrm{sec}$. The PCR products were assayed by a dissociation curve to verify a single product generation at the end point of the assay.

Western blot analysis. The cells were lysed in radioimmunoprecipitation assay (RIPA) buffer on ice and centrifuged at $12,000 \mathrm{x} \mathrm{g}$ for $30 \mathrm{~h}$ to obtain the supernatant. The extracted protein samples were separated by $12 \%$ SDS-PAGE and transferred onto polyvinylidene difluoride (PVDF) membranes (GE Healthcare, Amersham, UK). The membranes were blocked in 5\% skimmed milk for $1 \mathrm{~h}$ and subsequently incubated with primary antibodies at $4^{\circ} \mathrm{C}$ overnight. Following washing with PBS three times, the samples were probed by secondary antibodies conjugated with horseradish peroxidase for $1 \mathrm{~h}$ at room temperature. The signals were detected using a chemiluminescence system SuperSignal West Pico Chemiluminescent Substrate (Pierce Biotechnology, Inc., Rockford, IL, USA). The three independent experiments were repeated to assess the relative protein levels.

Cell invasion assay. The cell invasion assay was performed in 24-well FluoroBlok cell culture inserts with $8-\mu \mathrm{m}$ poresize polyethylene terephthalate (PET) membranes (BD Biosciences, Franklin Lakes, NJ, USA). The insert was coated with $200 \mu \mathrm{l}$ of $1 \mu \mathrm{g} / \mu \mathrm{l}$ Matrigel matrix (BD Biosciences) at $4^{\circ} \mathrm{C}$ overnight. Following starvation for $6 \mathrm{~h}$ in serum-free DMEM, the cells were harvested from one subconfluent $10 \mathrm{~cm}$ dish by cell dissociation buffer (Invitrogen Life Technologies), centrifuged at $300 \mathrm{x}$ gh for $5 \mathrm{~min}$ and resuspended in DMEM. Cells $\left(1 \times 10^{5}\right.$, in $500 \mu$ DMEM) were seeded onto the insert and $250 \mu \mathrm{l}$ DMEM with 10\% FBS was added into the lower chamber of the transwells. Following incubation for $18 \mathrm{~h}$ at $37^{\circ} \mathrm{C}$, the medium inside the insert was removed and the insert was then placed in a novel 24-well plate. The invaded cells on the reverse side of the insert were labeled with a fluorescent dye, calcein acetoxymethl ester ( $4 \mu \mathrm{M}$ in PBS; BD Biosciences), for $1 \mathrm{~h}$ at $37^{\circ} \mathrm{C}$. The fluorescence was measured with $494 / 517 \mathrm{~nm}$ (excitation/emission wavelength) by a $\mathrm{DU}^{\circledR}-8$ UV-Vis spectrophotometer (Beckman Coulter, Miami, FL, USA).

Statistical analysis. Data are presented as the mean \pm standard error of the mean of independent experiments. One-way analysis of variance (ANOVA) was used to determine the differences among the groups. The normality and constant variance for experimental data were tested by the Levene's test. Data without homogenous variance were log-transformed to meet the necessary assumptions of the analysis of variance. $\mathrm{P}<0.05$ was considered to indicate a statistically significant 
Table I. Correlation between TRB3 expression and clinicopathological factors in the 60 patients with NSCLC.

\begin{tabular}{|c|c|c|c|}
\hline \multirow[b]{2}{*}{ Characteristic } & \multicolumn{3}{|c|}{ TRB3 expression } \\
\hline & Low (0 and 1$)$ & $\operatorname{High}(2$ and 3$)$ & P-value ${ }^{a}$ \\
\hline \multicolumn{4}{|l|}{ Age } \\
\hline Years, mean \pm SD & $61.5 \pm 5.1$ & $60.87 \pm 7.5$ & 0.4782 \\
\hline \multicolumn{4}{|l|}{ Gender } \\
\hline Male & 16 & 17 & 0.5712 \\
\hline Female & 13 & 14 & \\
\hline \multicolumn{4}{|l|}{ Smoking status } \\
\hline Smoker & 17 & 15 & 0.8575 \\
\hline Non-smoker & 12 & 16 & \\
\hline \multicolumn{4}{|l|}{ Histological type } \\
\hline Adenocarcinoma & 14 & 21 & 0.0280 \\
\hline Squamous cell carcinoma & 20 & 10 & \\
\hline Large cell carcinoma & 3 & 5 & \\
\hline \multicolumn{4}{|l|}{ Stage } \\
\hline I and II & 18 & 10 & 0.0352 \\
\hline III and IV & 11 & 21 & \\
\hline \multicolumn{4}{|l|}{ Tumor status } \\
\hline $\mathrm{T} 1$ and $\mathrm{T} 2$ & 14 & 17 & 0.2782 \\
\hline T3 and T4 & 15 & 14 & \\
\hline \multicolumn{4}{|l|}{ Lymph node metastasis } \\
\hline N0 & 18 & 9 & 0.0168 \\
\hline N1-N3 & 11 & 21 & \\
\hline \multicolumn{4}{|l|}{ Distal metastasis status } \\
\hline M0 & 19 & 9 & 0.0316 \\
\hline M1 & 10 & 21 & \\
\hline \multicolumn{4}{|l|}{ Recurrence status } \\
\hline Yes & 11 & 23 & 0.0013 \\
\hline No & 18 & 7 & \\
\hline
\end{tabular}

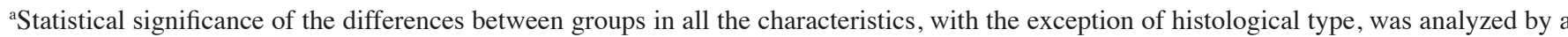
two-sided Fisher's exact test. The P-value for histological type was analyzed by the $\chi^{2}$ test. TRB3, tribbles homolog 3 ; NSCLC, non-small cell lung cancer.

difference. When the $\mathrm{F}$ value exceeded the critical value $(\mathrm{P}<0.05)$, the Newman-Keuls post hoc test was performed to compare the groups.

\section{Results}

Elevated expression of TRB3 in lung cancer. We assessed the differential expression of TRB3 in lung cancer specimens. The mRNA levels of TRB3 in tumor lesions of patients with non-small cell lung cancer (NSCLC) were determined by Q-PCR. Fifty-six of the sixty tumor samples showed a higher expression level of TRB3 compared with their respective adjacent, normal tissues (Fig. 1A). The upregulation of TRB3 was associated with distal metastasis and disease recurrence (Table I). Furthermore, the Kaplan-Meier survival curves revealed that TRB3 expression was inversely correlated with overall survival (Fig. 1B) and disease-free survival (Fig. 1C).
The IHC results demonstrated that TRB3 was upregulated in the two types of NSCLC investigated, particularly in adenocarcinoma; however, TRB3 upregulation was not observed in normal lung tissues (Fig. 1D). The TRB3 expression was significantly correlated with tumor size and lymph node or distal metastasis status in NSCLC patients (Table I). These results suggested that the upregulation of TRB3 correlated with poor prognosis in patients with NSCLC.

TRB3 knockdown results in apoptosis in human lung adenocarcinoma cells. To elucidate the effect of knocking down TRB3 on human lung adenocarcinoma cells, A549 cells were transfected with a TRB3 interference vector and their proliferation and characteristics were detected. The expression level of TRB3 was significantly lower following shTRB3 transfection compared with the control groups (Fig. 2A). Additionally, the knockdown of TRB3 exhibited a positive effect on cell 

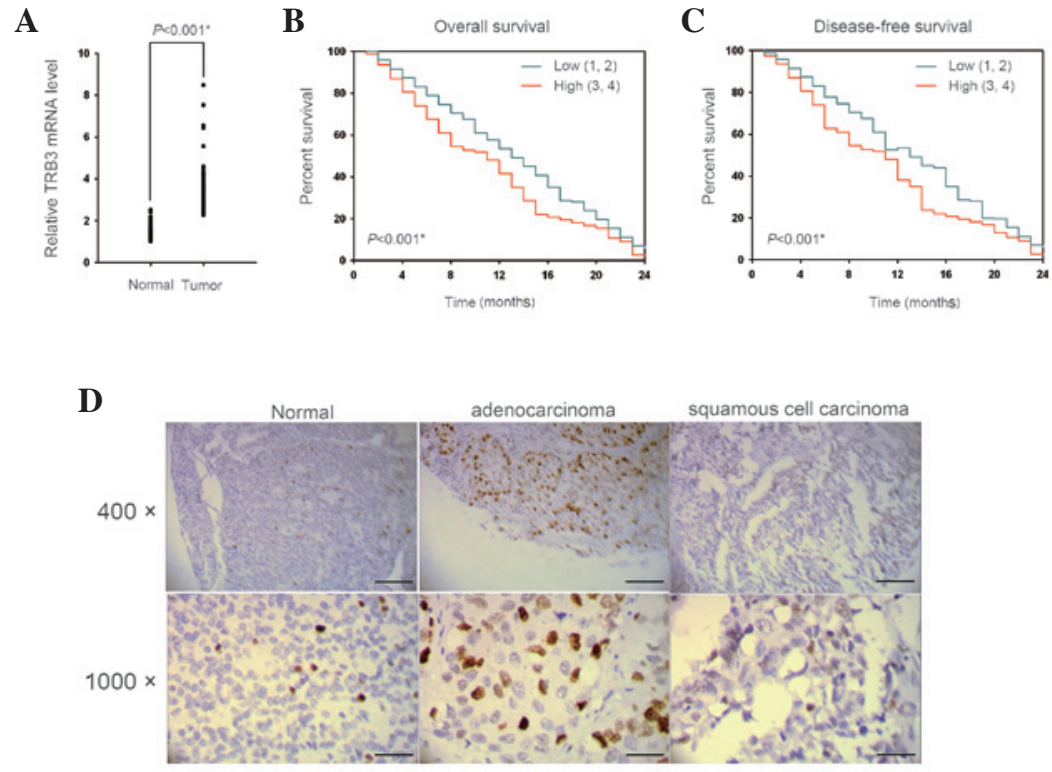

Figure 1. TRB3 expression in NSCLC and its correlation with survival rates. (A) Transcripts levels of TRB3 mRNA expression in normal and tumor tissues. (B) Overall survival and (C) disease-free survival in low (relative expression, 1 and 2) and high (relative expression, 3 and 4) TRB3 expression levels. (D) Immunohistochemistry of TRB3 in different types of lung cancer tissues (adenocarcinoma and squamous cell carcinoma) as well as in normal lung tissue. TRB3; tribbles homolog 3; NSCLC, non-small cell lung cancer.

A

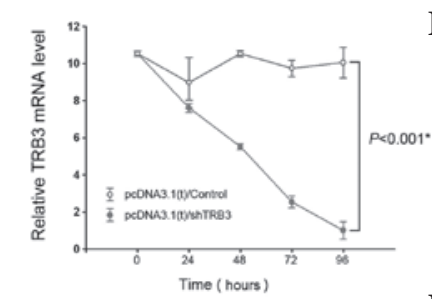

C

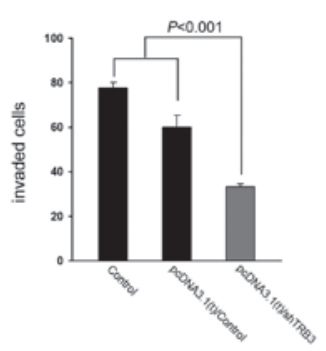

B

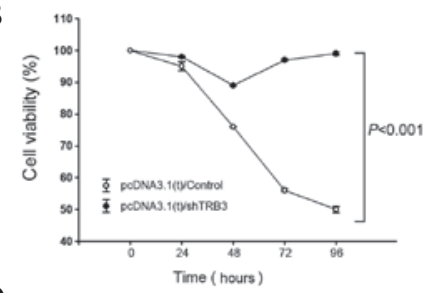

D

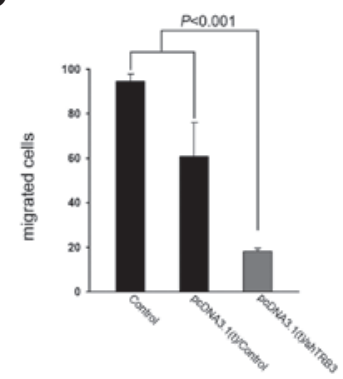

Figure 2. Cell biological activites following transfection of A549 cells with either the shTRB3 vector or empty vector control. (A) TBR3 mRNA levels and (B) cell viability at different time points following vector transfection. (C) Invaded cells and (D) migrated cells following vector transfection. TRB3; tribbles homolog 3; shTRB3, short hairpin-tribbles homolog 3 .

A

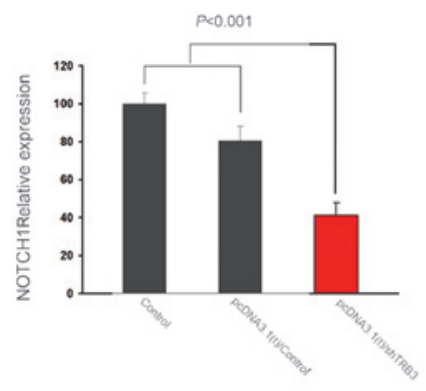

B

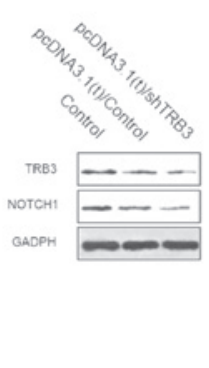

C

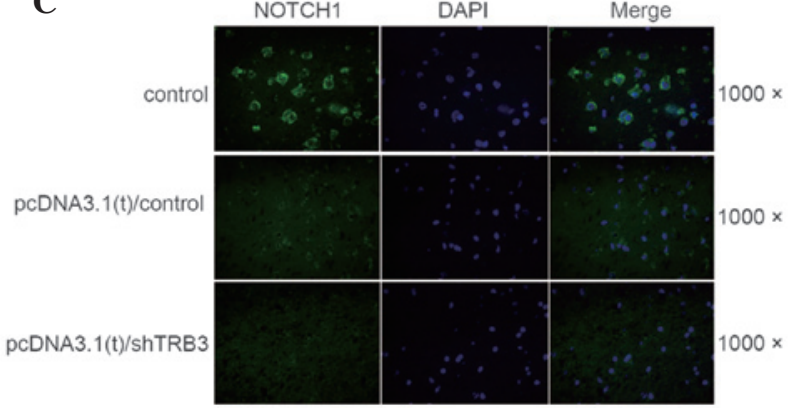

Figure. 3 Correlation between Notch 1 and TRB3 expression in A549 cells transfected with either the shTRB3 vector or the empty control vector. NOTCH1 is downregulated following shTRB3 vector transfection at the mRNA and protein levels by (A) real time PCR; (B) western blot analysis and (C) immunofluorescence, respectively. TRB3, tribbles 3 homolog; shTRB3, short hairpin-tribbles homolog 3 . 
growth (Fig. 2B). The shTRB3 groups had the lowest transwell level compared with the remaining groups.

To understand whether TRB3 is biologically significant in the aggressiveness of lung cancer cells, A549 cells were subjected to TRB3 knockdown and examined for their aggressiveness in vitro. Knockdown of TRB3 was revealed to significantly decrease the invasive and migratory abilities of the A549 cells (Fig. 2C and D, respectively).

Correlation between TRB3 and Notch expression in the lung adenocarcinoma cell lines. Following determination of the elevated expression of TRB3 in lung cancer and the apoptotic effect of the TRB3 knockdown on the adenocarcinoma cells, the underlying mechanism of this effect was investigated. The results demonstrated a positive correlation between TRB3 and Notch 1 expression, at both the gene and protein level, in the lung adenocarcinoma cell lines (Fig. 3).

\section{Discussion}

The tribbles gene family was initially identified in Drosophila and considered as an inhibitor of mitosis that regulates cell proliferation, migration and morphogenesis during development $(27,28)$. The three tribbles homologs, TRB1, TRB2 and TRB3, are considered to be members of the pseudokinase family, which contain a Ser/Thr protein kinase-like domain; however, lack the ATP binding pocket and catalytic residues. TRB3 is the most widely studied member of the mammalian tribbles family. The molecules which interact with TRB3 include transcription factors, such as ubiquitin ligase and the BMP type II receptor, which are members of the MAPK and PI3K signaling pathways $(29,30)$. Hua et al demonstrated that TRB3 interacts with SMAD3 and promotes tumor cell migration and invasion (30). The authors suggested that TRB3 is a novel partner of SMAD3 and may be involved in retaining SMAD3 in the nucleus by physical interaction, and maintaining the mesenchymal status of tumor cells. These studies suggested that TRB3 may be a potential therapeutic target for the treatment of human tumor metastasis. In the present study, it was identified that TRB3 exhibits an abnormally abundant expression in lung cancer tissues in patients with NSCLC, and that the upregulation of TRB3 was correlated with an increased number of tumor metastases, a higher recurrence of tumors and poorer survival. According to these results, we hypothesized that TRB3 was a significant factor in promoting the malignant progression of tumors.

Our results demonstrated that suppressing TRB3 expression significantly inhibited tumor metastasis in A549 human lung adenocarcinoma cells. The knockdown of TRB3 affected cell growth and metastatic ability. The cells transfected with shTRB3 remained in the G1 stage compared with those in the non-treated group. This suggested that following the suppression of TRB3, the cell cycle was altered to remain in the G1 stage, as opposed to passing into the $\mathrm{S}$ and $\mathrm{G} 2$ stages. In addition, the invasive ability of the A549 cells was significantly decreased following the suppression of TRB3 expression. There are a limited number of studies concerned with the effects of TRB3 on the cell cycle regardless of the fact that studies in neuronal PC6-3 cells have demonstrated that TRB3 is involved in neuronal apoptosis evoked by nerve growth factor withdrawal. TRB3 is also a multi-functional adaptor in a number of signaling pathways (31). For example, TRB3 has been demonstrated to inhibit insulin-induced S6 kinase activation (31). Furthermore, it has been revealed that TRB3 binds to ATF4 and regulates its transcriptional activity (32). The expression of TRBs is regulated by inflammatory stimulation and is cell type specific (33). TRB3 mRNA may be upregulated by various stresses. Also, TRB3 is the transcriptional target of several factors, including PPAR $\alpha$, ATF4-CHOP and PI-3K (34-36). These studies indicated that TRB3 participates in multiple cellular processes and pathways.

JAG1/Notch signaling is a mediator of cancer progression and metastasis, which is associated with the basal-like cancer subtype (17). Therefore, the components of the Notch 1 pathway are attractive therapeutic targets for this cancer subtype. Whether TRB3 affects tumor cell biology may be inferred from data concerning the pathways it regulates. Tumor-initiating cells (TIC), a small population of cells within certain types of tumors, are able to produce derivatives that maintain the tumor. The pathways that TRB3s target, such as the Notch pathway, have been implicated in TIC maintenance. This suggests that through the control of these pathways, TRB3 may regulate tumor initiation. The metastatic potential of epithelial tumors likely depends on a process known as EMT, where epithelial cells acquire a migratory mesenchymal phenotype. The Notch 1 pathways interact with each other and have a synergistic effect on the production of factors that promote EMT and metastasis (24). In addition, the locations of metastases have been shown to be influenced by the Notch pathways (37). It has been demonstrated that the release of TGF $\beta$ stimulates JAG1 expression in tumor cells and enhances Notch activation in osteoblasts and preosteoclasts, which may promote bone invasion (38). Collectively, these findings predict that TRB3 may potentiate the initiation of tumor formation and the metastatic capacity of cancer cells, through its regulation of JAG1/Notch activation. However, whether the activation of these pathways and processes are regulated by TRB3, and whether reduced survival time is associated with tumors with an elevated level of TRB3, have not yet been identified. In this study, we demonstrated that Notch 1 gene expression was positively correlated with TRB3 in A549 cells.

The current study indicated that TRB3 expression was elevated in lung cancer tissues in patients with NSCLC. In addition, loss of TRB3 induced an apoptotic effect in the A549 lung adenocarcinoma cell lines. The cell cycles were held in the G1 stage and the invasive ability of the cells decreased significantly. The results also identified a positive correlation between TRB3 and Notch 1 in the A549 cells. Thus, this interaction may provide potential therapeutic targets for human NSCLC.

\section{Acknowledgements}

This study was supported by funding from the Hunan Provincial Department of Science and Technology (grant no. 2012SK3249) and the Hunan Provincial Department of Health (grant no. B2012-098), China.

\section{References}

1. Boudeau J, Miranda-Saavedra D, Barton GJ and Alessi DR: Emerging roles of pseudokinases. Trends Cell Biol 16: 443-452, 2006. 
2. Izrailit J, Berman HK, Datti A, Wrana JL and Reedijk M: High throughput kinase inhibitor screens reveal TRB3 and MAPK-ERK/TGF $\beta$ pathways as fundamental Notch regulators in breast cancer. Proc Natl Acad Sci USA 110: 1714-1719, 2013.

3. Raja E: Cross-regulation between TGF $\beta / B M P$ Signalling and the metabolic LKB1 pathway. Doctoral thesis, Uppsala University, 2012.

4. Bowers AJ, Scully S and Boylan JF: SKIP3, a novel Drosophila tribbles ortholog, is overexpressed in human tumors and is regulated by hypoxia. Oncogene 22: 2823-2835, 2003.

5. Du K, Herzig S, Kulkarni RN and Montminy M: TRB3: a tribbles homolog that inhibits Akt/PKB activation by insulin in liver. Science 300: 1574-1577, 2003.

6. Wennemers M, Bussink J, Scheijen B, et al: Tribbles homolog 3 denotes a poor prognosis in breast cancer and is involved in hypoxia response. Breast Cancer Res 13: R82, 2011.

7. Miyoshi N, Ishii $\mathrm{H}$, Mimori K, et al: Abnormal expression of TRIB3 in colorectal cancer: a novel marker for prognosis. Br J Cancer 101: 1664-1670, 2009

8. Reedijk M, Odorcic S, Chang L, et al: High-level coexpression of JAG1 and NOTCH1 is observed in human breast cancer and is associated with poor overall survival. Cancer Res 65: 8530-8537, 2005.

9. Lee CW, Raskett CM, Prudovsky I and Altieri DC: Molecular dependence of estrogen receptor-negative breast cancer on a notch-survivin signaling axis. Cancer Res 68: 5273-5281, 2008.

10. Leong KG, Niessen K, Kulic I, et al: Jagged1-mediated Notch activation induces epithelial-to-mesenchymal transition through Slug-induced repression of E-cadherin. J Exp Med 204 2935-2948, 2007.

11. Rustighi A, Tiberi L, Soldano A, et al: The prolyl-isomerase Pin1 is a Notch1 target that enhances Notch1 activation in cancer. Nat Cell Biol 11: 133-142, 2009.

12. Shimizu M, Cohen B, Goldvasser P, Berman H, Virtanen C and Reedijk M: Plasminogen activator $\mathrm{UPA}$ is a direct transcriptional target of the JAG1-Notch receptor signaling pathway in breast cancer. Cancer Res 71: 277-286, 2011.

13. Honjo T: The shortest path from the surface to the nucleus: RBP-J kappa/Su(H) transcription factor. Genes Cells 1: 1-9, 1996.

14. Weijzen S, Rizzo P, Braid M, et al: Activation of Notch-1 signaling maintains the neoplastic phenotype in human Ras-transformed cells. Nat Med 8: 979-986, 2002.

15. Pece S, Serresi M, Santolini E, et al: Loss of negative regulation by Numb over Notch is relevant to human breast carcinogenesis. J Cell Biol 167: 215-221, 2004.

16. Colaluca IN, Tosoni D, Nuciforo P, et al: NUMB controls p53 tumour suppressor activity. Nature 451: 76-80, 2008.

17. Lee CW, Simin K, Liu Q, et al: A functional Notch-survivin gene signature in basal breast cancer. Breast Cancer Res 10: R97, 2008.

18. Yamaguchi N, Oyama T, Ito E, et al: NOTCH3 signaling pathway plays crucial roles in the proliferation of ErbB2-negative human breast cancer cells. Cancer Res 68: 1881-1888, 2008.

19. Dent R, Trudeau M, Pritchard KI, et al: Triple-negative breast cancer: clinical features and patterns of recurrence. Clin Cancer Res 13: 4429-4434, 2007.
20. Farnie G and Clarke RB: Mammary stem cells and breast cancer - role of Notch signalling. Stem Cell Rev 3: 169-175, 2007.

21. Yin X, Wolford CC, Chang YS, et al: ATF3, an adaptive-response gene, enhances TGF $\{$ beta\} signaling and cancer-initiating cell features in breast cancer cells. J Cell Sci 123: 3558-3565, 2010.

22. Kalluri R and Weinberg RA: The basics of epithelial-mesenchymal transition. J Clin Invest 119: 1420-1428, 2009.

23. Gavert N and Ben-Ze'ev A: Epithelial-mesenchymal transition and the invasive potential of tumors. Trends Mol Med 14: 199-209, 2008.

24. Timmerman LA, Grego-Bessa J, Raya A, et al: Notch promotes epithelial-mesenchymal transition during cardiac development and oncogenic transformation. Genes Dev 18: 99-115, 2004

25. Xie L,Law BK, Chytil AM, Brown KA, Aakre ME and Moses HL: Activation of the Erk pathway is required for TGF-beta1-induced EMT in vitro. Neoplasia 6: 603-610, 2004.

26. Sethi N, Dai X, Winter CG and Kang Y: Tumor-derived JAGGED1 promotes osteolytic bone metastasis of breast cancer by engaging notch signaling in bone cells. Cancer cell 19: 192-205, 2011.

27. Grosshans J and Wieschaus E: A genetic link between morphogenesis and cell division during formation of the ventral furrow in Drosophila. Cell 101: 523-531, 2000.

28. Seher TC and Leptin M: Tribbles, a cell-cycle brake that coordinates proliferation and morphogenesis during Drosophila gastrulation. Curr Biol 10: 623-629, 2000

29. Sieber C, Kopf J, Hiepen C and Knaus P: Recent advances in BMP receptor signaling. Cytokine Growth Factor Rev 20: 343-355, 2009.

30. Hua F, Mu R, Liu J, et al: TRB3 interacts with SMAD3 promoting tumor cell migration and invasion. J Cell Sci 124: 3235-3246, 2011.

31. Matsushima R, Harada N, Webster NJ, Tsutsumi YM and Nakaya Y: Effect of TRB3 on insulin and nutrient-stimulated hepatic p70 S6 kinase activity. J Biol Chem 281: 29719-29729, 2006.

32. Izrailit J, Berman HK, Datti A, Wrana JL and Reedijk M: High throughput kinase inhibitor screens reveal TRB3 and MAPK-ERK/TGF $\beta$ pathways as fundamental Notch regulators in breast cancer. Proc Natl Acad Sci USA 110: 1714-1719, 2013.

33. Xu J, Lv S, Qin Y, et al: TRB3 interacts with CtIP and is overexpressed in certain cancers. Biochim Biophys Acta 1770: 273-278, 2007.

34. Schwarzer R, Dames S, Tondera D, Klippel A and Kaufmann J: TRB3 is a PI 3-kinase dependent indicator for nutrient starvation. Cell Signal 18: 899-909, 2006.

35. Koo SH, Satoh H, Herzig S, et al: PGC-1 promotes insulin resistance in liver through PPAR-alpha-dependent induction of TRB-3. Nat Med 10: 530-534, 2004

36. Ohoka N, Yoshii S, Hattori T, Onozaki K and Hayashi H: TRB3, a novel ER stress-inducible gene, is induced via ATF4-CHOP pathway and is involved in cell death. EMBO J 24: 1243-1255, 2005.

37. Valastyan S and Weinberg RA: Tumor metastasis: molecular insights and evolving paradigms. Cell 147: 275-292, 2011.

38. Weilbaecher KN, Guise TA and McCauley LK: Cancer to bone: a fatal attraction. Nat Rev Cancer 11: 411-425, 2011. 